\title{
Performance of English stop smoking services in first 10 years: analysis of service monitoring data
}

\author{
(c) $\stackrel{(1)(8)}{\text { By }}$ OPEN ACCESS
}

\author{
Robert West professor of health psychology ${ }^{12}$, Sylvia May consultant researcher ${ }^{2}$, Matthew West \\ consultant researcher ${ }^{2}$, Emma Croghan director of public health and lifestyle services ${ }^{3}$, Andy \\ McEwen senior research associate ${ }^{12}$
}

${ }^{1}$ Cancer Research UK Health Behaviour Research Centre, Department of Epidemiology and Public Health, University College London, London WC1E 6BT, UK; ${ }^{2}$ National Centre for Smoking Cessation and Training (NCSCT), 1-6 Yarmouth Place, London W1J 7BU, UK; ${ }^{3}$ North 51 Ltd, Biocity, Nottingham, UK

\begin{abstract}
Objectives To analyse the performance of the English Stop Smoking Services from 2001/02 to 2010/11.

Design Analysis of national service monitoring data.

Setting England.

Participants Smokers recorded as having been treated by English stop smoking services between April 2001 and March 2011.

Main outcome measures Annual figures for the number of quit dates set (throughput), the percentage of these that led to biochemically verified abstinence after four weeks (four week quit rate), and the "impact" in terms of the number of four week quitters beyond those who it is estimated would have stopped with only a prescription for smoking cessation treatment; characteristics of smokers being treated, medication used, and mode of delivery (for example, one to one, group based); variability across local services in throughput, four week quit rates, and impact for 2010/11.

Results Throughput rose from 227335 in 2001/02 to 787527 (8\% of all smokers) in 2010/11. The percentage of four week quitters declined slightly from $35 \%$ to $34 \%$. Impact rose from 22933 four week quitters created in 2001/02 to 72411 in 2010/11 (corresponding to an estimated 2172312 month quitters). The services were successful in reaching disadvantaged smokers; $54 \%$ ( $n=425684)$ were in receipt of free prescriptions in $2010 / 11$. Substantial variation existed across local services in throughput, success rates, and impact.

Conclusions The English stop smoking services have had an increasing impact in helping smokers to stop in their first 10 years of operation and have successfully reached disadvantaged groups. However, performance across local services has varied considerably.
\end{abstract}

\section{Introduction}

Stopping smoking after early adulthood adds an average of three months' healthy life expectancy for every year of smoking avoided, but for most smokers quitting is extremely difficult, with fewer than $5 \%$ of unaided quit attempts lasting 12 months. ${ }^{12}$ Evidence from multiple randomised controlled trials shows that face to face behavioural support for smoking cessation (also referred to as counselling and psychological support) provided individually or in groups can improve cessation rates and save lives very cheaply. ${ }^{34}$ The English National Health Service provides this support on top of medication to aid cessation. This takes the form of a network of "stop smoking services." Other countries have since followed this model for helping their smokers to stop. ${ }^{5}$ Extending global implementation of Article 14 of the World Health Organization Framework Convention on Tobacco Control, which focuses on treatment for tobacco dependence, and changes in funding arrangements in England, make it timely to examine the effect of the English stop smoking services. ${ }^{6}$ This paper reports an evaluation of 10 years of operation of these services.

In 1998 the UK government published a white paper entitled "Smoking Kills," outlining its plans to reduce the massive toll of death and disease in the country caused by smoking. ${ }^{7}$ It included a range of evidence based policies such as tax increases and use of mass media campaigns. One innovative component of these plans was establishing a national network of stop smoking services to ensure that every smoker in the country who wanted help with stopping would have access to evidence based behavioural support on top of a prescription for a smoking cessation medication, as recommended in clinical practice guidelines. $^{8}$

The stop smoking services in England are under local direction. Until 2013, this was through 151 primary care trusts; since April 2013, it has been through local authorities. Each local area can configure its service as it sees fit but is encouraged to follow national guidance. ${ }^{9}$ Services are expected to offer behavioural support and medication to all smokers in their community and 
also to ensure that they are treating smokers proportionally to their demographics in their area (for example, if $60 \%$ of the local smoking population is from disadvantaged groups, the service will be expected to treat at least that proportion of disadvantaged smokers).

The behavioural support should involve a session before the quit date in which smokers discuss medication options and preparation for the quit date, a quit date session, and then weekly sessions for at least four weeks afterwards. The sessions seek to maintain motivation to sustain abstinence, provide advice on avoiding or coping with cigarette cravings and adverse withdrawal symptoms, and optimise use of medication. ${ }^{9}$ Medication should be offered for at least eight weeks in the case of nicotine replacement therapy and 12 weeks in the case of varenicline. ${ }^{8}$ Evidence from randomised controlled trials and an early evaluation of the services indicates that when these are provided optimally, the proportion of users who stop for four weeks should be approximately 50\%, with $15 \%$ lasting 12 months, compared with $15 \%$ at four weeks and less than $5 \%$ at 12 months if these smokers tried to stop unaided. ${ }^{1}{ }^{10} 11$

The services are free to users. A small prescription charge for medications is payable; people on low incomes, over 60 years of age, or pregnant/postpartum and patients with certain medical conditions are exempt.

From the outset, each local stop smoking service has been required to submit quarterly data to the Department of Health on numbers of quit dates set (throughput), end of treatment abstinence rates (four weeks after the target quit date), characteristics of smokers using the services, and type of treatment provided. ${ }^{12}$ These data provide a resource for evaluating the stop smoking services and for examining trends over time.

When estimating the impact of any stop smoking treatment programme, one must take account of the fact that a proportion of the smokers attending would have stopped without support or with a lower level of support. This requires use of a comparator; in the case of the English stop smoking services, the most appropriate comparator involves assuming that all those attending the services would instead have tried to stop with a prescription for a smoking cessation medication but without the specialist behavioural support package. This is conservative because it focuses only on the behavioural support element (not the medication) and takes no account of possible effects that having services may have on promoting quit attempts. However, it permits assessment of the specific effect of having these services as opposed merely to providing stop smoking medication on prescription.

Research from numerous randomised controlled trials and cohort studies shows that $70 \%$ of people who are abstinent at four weeks will relapse by 12 months, ${ }^{10}$ and up to $35 \%$ of those remaining will relapse after that. ${ }^{13}$ With that information and knowledge of the background quit rates in the population, we can estimate the numbers of permanent ex-smokers generated by the services from the four week quit rates. ${ }^{13}$

This analysis attempted to answer the following research questions. How have throughput, success rates, and impact of the English stop smoking services changed in the past 10 years of their operation? To what extent have the services met the needs of different sub-populations and particularly economically disadvantaged smokers? How far have throughput, four week quit rates, and impact varied between local services?

\section{Methods}

We used mandatory monitoring data collected by the Health and Social Care Information Centre to do the analyses reported in this paper. ${ }^{12}$ We analysed service data for every year since 2001/02, when the services were implemented nationwide with full access to stop smoking medication.

"Treated smokers" included in the returns were service attenders who had set a quit date and received an offer of a structured, multi-session intervention (delivered by a stop smoking adviser). An individual smoker may be treated more than once and so may appear in the data on more than one occasion in a single year or over several years. However, the vast majority of those treated in a year are "unique users." Analysis of a representative sample of 70 services using the Quit Manager database for $2010 / 11$ showed that $92 \%$ of treated smokers were counted only once that year, $7 \%$ were counted twice, and $1 \%$ were counted three times (E Croghan, personal communication, July 2012).

The data collected for each treated smoker included sex, age, ethnic group, eligibility for free prescriptions (from 2008/09), pregnancy, and type of medication used (none, nicotine replacement therapy, bupropion, and varenicline). From 2008/09 the mode of delivery of the behavioural support was recorded: closed group (same group of smokers seen together regularly), open/rolling group (smokers at different stages of their quit attempt, regular meetings offered), one to one support (one adviser, one smoker; specified time and place), couple/family support (adviser and up to six members of same

family/friendship group), drop-in support (one to one, specified venue but unallocated time within time slot), telephone support (one to one support over the phone), and "other" (for example, text messaging or internet based support).

Outcome was recorded in terms of whether a quit attempt resulted in a biochemically verified four week quitter. Four weeks after the target quit date (usually at the last treatment session), service users were asked whether they had smoked at all in the previous two weeks. They were thus allowed a two week grace period to get their quit attempt started. If they indicated that they had not smoked at all, good practice required the stop smoking adviser to take an expired air carbon monoxide reading. ${ }^{9}$ If the reading was below $10 \mathrm{ppm}$, the four week quit status would be regarded as confirmed. Lower carbon monoxide thresholds have been proposed, but research shows that these do not substantially affect the results. ${ }^{14}$ As is standard practice in smoking cessation, ${ }^{15}$ service users who did not return for the four week session or for whom an expired air carbon monoxide reading was not available were counted as having resumed smoking.

Another option in terms of calculating the four week quit rate would have been to use the number of people who self reported as having stopped at four weeks irrespective of whether the claim was biochemically verified. However, this would have provided an inflated estimate given the known degree of misreporting in these situations.

We defined the "impact" of the stop smoking services as the number of four week quitters generated over and above those that would have been expected had the same smokers received only a prescription for a stop smoking medication. The purpose of the measure was to provide the most accurate assessment of the effect of the behavioural support provided by the service.

A figure of $25 \%$ was arrived at as the best estimate for the expected biochemically verified four week quit rate with prescription medication alone. This figure was based on the four week success rate in smokers trying to stop unaided multiplied 
by the rate ratio of 1.6 attributable to medications from Cochrane reviews. ${ }^{16}$ Thus smokers in control conditions of smoking cessation trials achieve four week quit rates of approximately $15 \%$. Nicotine replacement therapy (the most popular medication) to aid cessation increases this by approximately $60 \%$, yielding a predicted four week quit rate of $25 \%$ for smokers motivated to seek help with quitting and using only nicotine replacement therapy. Using this information, we calculated impact as the difference between the achieved four week quit rate and $25 \%$, multiplied by the number of quit dates set.

\section{Results}

Annual throughput increased from 227335 quit dates set in 2001/02 to 787527 in 2010/11 (table $1 \Downarrow$; fig $1 \Downarrow$ ). The quit rate reduced slightly from $35 \%$ to $34 \%$, with a dip to $31 \%$ in $2007 / 08$. This meant that the total number of four week quitters rose from 79767 to 269293 . The impact (number of four week quitters estimated to be created beyond what would be expected with medication alone) rose from 22933 to 72411 .

Table $2 \Downarrow$ shows that the services attracted an increasing proportion of men and primarily served smokers aged over 35 , and that the proportion of smokers from ethnic minority groups who attended almost doubled to $7 \%$. The proportion of smokers eligible for free prescriptions rose considerably between 2008/09 and 2010/11 until it comprised more than half of those treated.

Table $3 \Downarrow$ shows the types of medication provided. Throughout the period of study, nicotine replacement therapy was the dominant medication. Initially, bupropion was also used by a substantial minority; by 2010/11, this had been replaced by varenicline, which became available in $2007 / 08$. Table $4 \Downarrow$ shows that by far the most common mode of behavioural support was one to one support.

Figure $2 \Downarrow$ shows the variation in throughput, four week quit rates, and impact of the 151 local stop smoking services in 2010/11. Throughput and impact are expressed per 100000 head of local population to take account of differences in size of primary care trusts. The trusts in each case are shown from highest to lowest, left to right. The order of the trusts is different for each outcome measure. In the case of impact, several primary care trusts were rated as zero because their four week quit rates were below $25 \%$.

We found a moderate negative correlation between four week quit rate (percentage of those treated who were abstinent at four weeks) and throughput (number of quit dates set) per 100000 head of population (Pearson $r=-0.45, \mathrm{P}<0.001$ ). Impact was highly correlated with four week quit rate (Pearson $r=0.86$, $\mathrm{P}<0.001$ ) but negatively correlated with throughput (Pearson $r=-0.26, \mathrm{P}<0.01)$.

\section{Discussion}

The English stop smoking services have had an increasing impact on national smoking cessation since 2001 by increasing throughput with only a small decrease in the percentage of those treated who succeed. They have been successful in reaching economically disadvantaged smokers, with more than half of those treated being eligible for free prescriptions. The quit rates of around 35\% at four weeks have been generally below what would be expected from an optimal service (around 50\%) but higher than would be expected if the smokers attending had received only a prescription for a stop smoking medication (25\%). We found substantial local variation in throughput, four week quit rates, and impact. Services with higher throughput have lower success rates, and impact is strongly associated with percentage of those treated who quit rather than with throughput.

Bearing in mind the difficulty in quitting experienced by smokers who typically attend stop smoking services, even a $35 \%$ four week quit rate represents a substantial impact when applied to the nearly 800000 quit dates set with the services in 2010/11 (700 000 smokers, or $8 \%$ of the 9 million smokers in England). Using an estimate of $70 \%$ relapse between four weeks and 12 months, the services generated 2172312 month quitters in 2010/11 (30\% of the four week impact). According to standard cost effectiveness tables developed for the purpose, ${ }^{13}$ this is expected to result in 24413 additional life years (discounted by $3.5 \%$ per year as recommended by the National Institute for Health and Care Excellence (NICE)). The estimated cost of the service in 2010/11 was £84m (€97m; \$128m) excluding medication costs ${ }^{9}-£ 107$ per quit date set, $£ 312$ per four week quitter, and $£ 3441$ per discounted life year saved, which is less than $20 \%$ of the NICE threshold.

The wide variation in throughput, four week quit rates, and estimated impact of the local services needs further investigation. Other research on a sample of local services suggests that it cannot be accounted for by variation in the characteristics of smokers in the local population. ${ }^{17}$ A substantial part of it can be attributed to differing degrees to which services follow evidence based practice, including use of specialist stop smoking advisers and ensuring that smokers have full access to optimum medication options. ${ }^{17}$ Thus, services that make greater use of group based support and specialist services delivered by staff employed specifically for the purpose, and who provide varenicline or combination nicotine replacement (transdermal patch plus a faster acting product), achieve the highest success rates. ${ }^{17}$ In addition, services that offer more sessions of support and where the behavioural support is more in line with what has been shown to be effective in randomised controlled trials get better success rates. ${ }^{18}$ The National Centre for Smoking Cessation and Training (NCSCT) was set up by the Department of Health in 2008 to reduce this variability by establishing best practice, implementing a training and assessment programme to ensure a minimum level of competence of stop smoking advisers (www.ncsct.co.uk).

As with any routine data monitoring, the findings reported here are subject to several potential sources of error and bias. Focus on four week quitters verified by carbon monoxide would lead to underestimation of effectiveness of services that have not followed guidance in attempting to ensure that all claims of abstinence are biochemically verified. This could be why several services had success rates of less than $25 \%$. Also, these services may not have been providing high quality support.

With strong pressure on primary care trusts to achieve four week quit targets, some services may also have adopted more lenient criteria when determining throughput and four week quit status (for example, counting smokers as having quit through the service when they were detected as a quitters only through questioning after the fact). The Department of Health has commissioned the NCSCT to develop a process for reviewing service provision and monitoring to mitigate variability in reporting practice. ${ }^{19}$ Several sensitivity analyses to establish a plausible range within which the likely effects of the services lie would be possible; however, even under pessimistic assumptions, the number of life years gained and cost effectiveness place them among the most impactful and cost effective parts of national healthcare provision. Finally, the figure for impact may be an underestimate if existence of stop smoking support has led more people to try to quit than would have done otherwise. 
Other countries that have national services, or regions that have regional ones, can be reassured that providing this kind of "individual level" public health intervention at a "population level" is possible. However, they can also learn lessons from the English services, documented in this paper, about the need to pay close attention to variability in performance, and the importance of rigorous monitoring and finding ways of fostering good practice.

\section{Conclusion}

Over 10 years of operation, the English stop smoking services have increased their reach and impact threefold. In 2010/11 they were used by some $8 \%$ of all smokers, including a high proportion of those with economic disadvantage, and can estimated to have helped more than 20000 to achieve long term abstinence, saving almost 25000 life years. However, considerable variability in outcomes exists across local areas.

Contributors: RW had the original idea for this paper, led the analyses, and drafted the manuscript. MW extracted data from the Information Centre publications and produced the figures. SM assisted with the drafting and data analyses. AMcE and EC made significant contributions to the interpretation of the results and the writing of the paper. All authors had full access to all of the data (including statistical reports and tables) in the study and can take responsibility for the integrity of the data and the accuracy of the data analysis. All authors reviewed and approved the final manuscript. RW is the guarantor.

Funding: This paper was part of the work of the National Centre for Smoking Cessation and Training that was funded by the Department of Health (DOH T336/BSS/M award number 49945). RW is a member of the UK Centre for Tobacco Control Studies, and RW and AMcE are funded by Cancer Research UK. The study sponsor had no role in the study design; the collection, analysis, and interpretation of data; the writing of the article; or the decision to submit it for publication. The researchers are independent from the funder.

Competing interests: All authors have completed the ICMJE uniform disclosure form at www.icmje.org/coi_disclosure.pdf (available on request from the corresponding author) and declare: all authors have support from NHS Centre for Smoking Cessation and Training (NCSCT) for the submitted work; RW is a director of the NCSCT, undertakes research and consultancy for companies that develop and manufacture smoking cessation medications (Pfizer, Johnson \&Johnson, McNeil, GlaxoSmithKline, Nabi, Novartis, and Sanofi-Aventis), has a share of a patent for a novel nicotine delivery device, and is a trustee of QUIT, a charity that provides stop smoking support; MW has a share of a patent for a novel nicotine delivery device. EC previously worked at the English Department of Health as the delivery lead for tobacco control policy, has received travel funding, honorariums, and consultancy payments from manufacturers of smoking cessation products (Pfizer, Johnson \&Johnson, McNeil, GlaxoSmithKline, Novartis, and

Sanofi-Aventis), and receives royalties from a book on smoking cessation and a book on health promotion. AMcE is a director of the NCSCT, has received travel funding, honorariums, and consultancy payments from manufacturers of smoking cessation products (Pfizer, GlaxoSmithKline, and Novartis), receives payment for providing training to smoking cessation specialists, receives royalties from books on smoking cessation, and has a share in a patent of a nicotine delivery device; no other relationships or activities that could appear to have influenced the submitted work.

Ethical approval: Not required for this study because it involved analysis of publically available data.

Data sharing: All stop smoking service and monitoring data can be obtained from the Health and Social Care Information Centre (http:// www.hscic.gov.uk/).

1 Hughes JR, Keely J, Naud S. Shape of the relapse curve and long-term abstinence among untreated smokers. Addiction 2004;99:29-38.

2 Doll R, Peto R, Boreham J, Sutherland I. Mortality in relation to smoking: 50 years observations on male British doctors. BMJ 2004;328:1519.

3 West R, McNeill A, Raw M. Smoking cessation guidelines for health professionals: an update. Thorax 2000;55:987-99.

4 National Institute for Health and Clinical Excellence. Smoking cessation services in primary care, pharmacies, local authorities and workplaces, particularly for manual working groups, pregnant women and hard to reach communities. NICE, 2008. (PH 10.)

5 Piné-Abata H, McNeill A, Murray R, Bitton A, Rigotti N, Raw M. A survey of tobacco dependence treatment services in 121 countries. Addiction 2013;108:1476-84

6 Piné-Abata H, McNeill A, Raw M, Bitton A, Rigotti N, Murray R. A survey of tobacco dependence treatment guidelines in 121 countries. Addiction 2013;108:1470-5.

7 Department of Health. Smoking kills: a white paper on tobacco. Stationery Office, 1999.

8 Raw M, McNeill A, West R. Smoking cessation guidelines for health professionals-a guide to effective smoking cessation interventions for the health care system. Thorax 1998;53:S1-19.

9 Department of Health. Local stop smoking services: key updates to the 2011/12 service delivery and monitoring guidance for 2012/13. Department of Health, 2013.

10 Ferguson J, Bauld L, Chesterman J, Judge K. The English smoking treatment services: one-year outcomes. Addiction 2005;100(suppl 2):59-69.

11 Stead LF, Lancaster T. Combined pharmacotherapy and behavioural interventions for smoking cessation. Cochrane Database Syst Rev 2012;(10):CD008286.

12 Health and Social Care Information Centre. Statistics on NHS stop smoking services-England, April 2010 to March 2011, annual report. 2011. www.hscic.gov.uk/ searchcatalogue? productid $=1253 \& q=$ title $\% 3 a \%$

22Statistics+on+NHS+Stop+Smoking+Services\%22\&sort=Relevance\&size=10\&page=1\# topNHC.

13 Stapleton JA, West R. A direct method and ICER tables for the estimation of the cost-effectiveness of smoking cessation interventions in general populations: application to a new cytisine trial and other examples. Nicotine Tob Res 2012;14:463-71.

14 Brose LS, Tombor I, Shahab L, West R. The effect of reducing the threshold for carbon monoxide validation of smoking abstinence-evidence from the English stop smoking services. Addict Behav 2013;38:2529-31.

15 West R, Hajek P, Stead L, Stapleton J. Outcome criteria in smoking cessation trials: proposal for a common standard. Addiction 2005;100:299-303.

16 Stead LF, Perera R, Bullen C, Mant D, Hartmann-Boyce J, Cahill K, et al. Nicotine replacement therapy for smoking cessation. Cochrane Database Syst Rev 2012;(11):CD000146.

17 Brose LS, West R, McDermott MS, Fidler JA, Croghan E, McEwen A. What makes for an effective stop-smoking service? Thorax 2011;66:924-6.

18 Michie S, Churchill S, West R. Identifying evidence-based competences required to deliver behavioral support for smoking cessation. Ann Behav Med 2011;41:59-70.

19 Mcllvar M, Williams I, McEwen A, West R. Development of an independent audit process for providers of stop smoking support. National Centre for Smoking Cessation and Training, 2012 (available at www.ncsct.co.uk/usr/pub/NCSCT-audit-report.pdf).

Accepted: 24 July 2013

\section{Cite this as: BMJ 2013;347:f4921}

This is an Open Access article distributed in accordance with the Creative Commons Attribution Non Commercial (CC BY-NC 3.0) license, which permits others to distribute, remix, adapt, build upon this work non-commercially, and license their derivative works on different terms, provided the original work is properly cited and the use is non-commercial. See: http://creativecommons.org/licenses/by-nc/3.0/. 


\section{What is already known on this topic}

The English stop smoking services have been successful in recruiting large numbers of smokers

\section{What this study adds}

This is the first published estimate of the impact of the English stop smoking services and overview of performance over its first 10 years of operation

It shows that the services have trebled their impact in terms of numbers of smokers led to stop by increasing the numbers treated with only a small decline in the success rates

However, substantial variability between local services is a matter that requires attention

\section{Tables}

Table 1| Throughput, number and percentage of four week quitters, and impact of English stop smoking services from 2001/02 to 2010/11

Year Throughput (No) No (\%) 4 week quitters Impact (No of 4 week quitters created)

\begin{tabular}{llll}
$2001 / 02$ & 227335 & $79767(35)$ & 22933 \\
\hline $2002 / 03$ & 234858 & $83163(35)$ & 24449 \\
\hline $2003 / 04$ & 361224 & $127493(35)$ & 37187 \\
\hline $2004 / 05$ & 529567 & $191025(36)$ & 58633 \\
\hline $2005 / 06$ & 603174 & $210717(35)$ & 59924 \\
\hline $2006 / 07$ & 600410 & $198052(33)$ & 47950 \\
\hline $2007 / 08$ & 680289 & $208742(31)$ & 38670 \\
\hline $2008 / 09$ & 671259 & $224278(33)$ & 56463 \\
\hline $2009 / 10$ & 757537 & $256713(34)$ & 67329 \\
\hline
\end{tabular}


Table 2| Characteristics of stop smoking service users. Values are numbers (percentages)

\begin{tabular}{llccc}
$\begin{array}{l}\text { Year } \\
2001 / 02\end{array}$ & Eligible for free prescriptions & Female & Minority ethnic group & Aged $\geq 35$ \\
\hline $2002 / 03$ & NA & $129101(57)$ & $7366(4)$ & $168173(74)$ \\
\hline $2003 / 04$ & NA & $134963(57)$ & $8252(4)$ & $166618(71)$ \\
\hline $2004 / 05$ & NA & $205768(57)$ & $14896(4)$ & $252659(70)$ \\
\hline $2005 / 06$ & NA & $302035(57)$ & $24012(5)$ & $367661(69)$ \\
\hline $2006 / 07$ & NA & $341295(57)$ & $32348(6)$ & $407100(68)$ \\
\hline $2007 / 08$ & NA & $334099(56)$ & $37035(6)$ & $401125(67)$ \\
\hline $2008 / 09$ & NA & $373000(55)$ & $37734(6)$ & $464825(68)$ \\
\hline $2009 / 10$ & $385509(44)$ & $357339(53)$ & $45228(7)$ & $464825(66)$ \\
\hline $2010 / 11$ & $425684(54)$ & $411392(52)$ & $57849(7)$ & $522037(66)$ \\
\hline
\end{tabular}

$\mathrm{NA}=$ not applicable 
Table 3| Percentage of quit attempts involving different types of stop smoking medication. Values are numbers (percentages)

\begin{tabular}{lllccc} 
Year & NRT & Bupropion & Varenicline & None & Other/not known \\
\hline $2001 / 02$ & $142260(63)$ & $44286(19)$ & NA & $24389(11)$ & $11887(5)$ \\
\hline $2002 / 03$ & $175674(75)$ & $26773(11)$ & NA & $19784(8)$ & $9140(4)$ \\
\hline $2003 / 04$ & $277041(77)$ & $30158(8)$ & NA & $27623(8)$ & $22611(6)$ \\
\hline $2004 / 05$ & $424509(80)$ & $33931(6)$ & NA & $35472(7)$ & $31473(6)$ \\
\hline $2005 / 06$ & $494105(82)$ & $30559(5)$ & NA & $40306(7)$ & $33814(6)$ \\
\hline $2006 / 07$ & $496932(83)$ & $27395(5)$ & NA & $38617(6)$ & $33656(6)$ \\
\hline $2007 / 08$ & $474311(70)$ & $22348(3)$ & $97259(14)$ & $42647(6)$ & $40977(6)$ \\
\hline $2008 / 09$ & $449876(67)$ & $11278(2)$ & $134949(20)$ & $35774(5)$ & $32445(6)$ \\
\hline $2009 / 10$ & $493459(65)$ & $9509(1)$ & $175380(23)$ & $39222(5)$ & $31093(5)$ \\
\hline $2010 / 11$ & $498399(63)$ & $6515(1)$ & $203338(26)$ & $39392(5)$ & $28557(4)$ \\
\hline
\end{tabular}

$\mathrm{NA}=$ not applicable; NRT=nicotine replacement therapy.

Row percentages may not add to 100 because of up to $2 \%$ receiving more than one medication, not recorded here. 
Table 4| Mode of service delivery. Values are numbers (percentages)

Year One to one Drop-in Rolling group Closed group Telephone Family/couple Other $2008 / 09526346(78) 63926(10) \quad 31089(5) \quad 18123(3) \quad 6920(1) \quad 4764(1) \quad 20088(3)$

$2009 / 10595629(79) 80448(11) \quad 33296(4) \quad 16680(2) \quad 9041(1) \quad 6268(1) \quad 16175(2)$

2010/11 $636036(81) 84743(11) \quad 26142(3) \quad 13899(2) \quad 11576(1) \quad 7822(1) \quad 7309(1)$




\section{Figures}

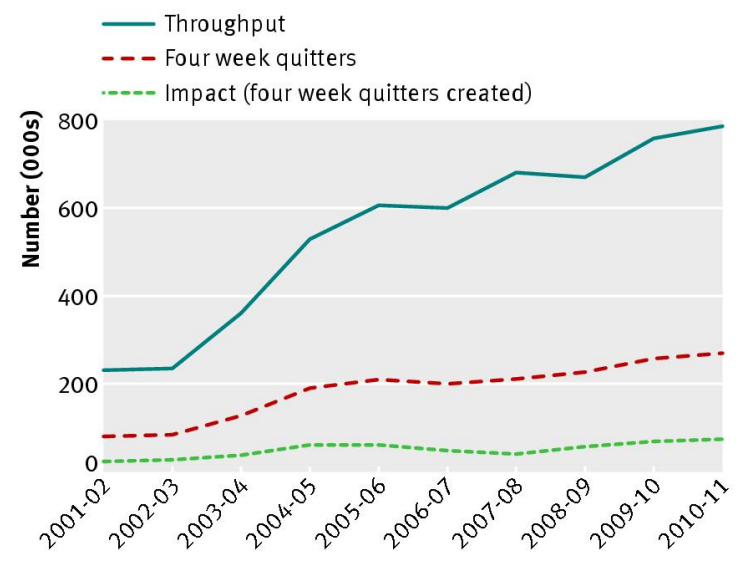

Fig 1 Annual number of quit dates set in English stop smoking services 2001/02 to 2010/11
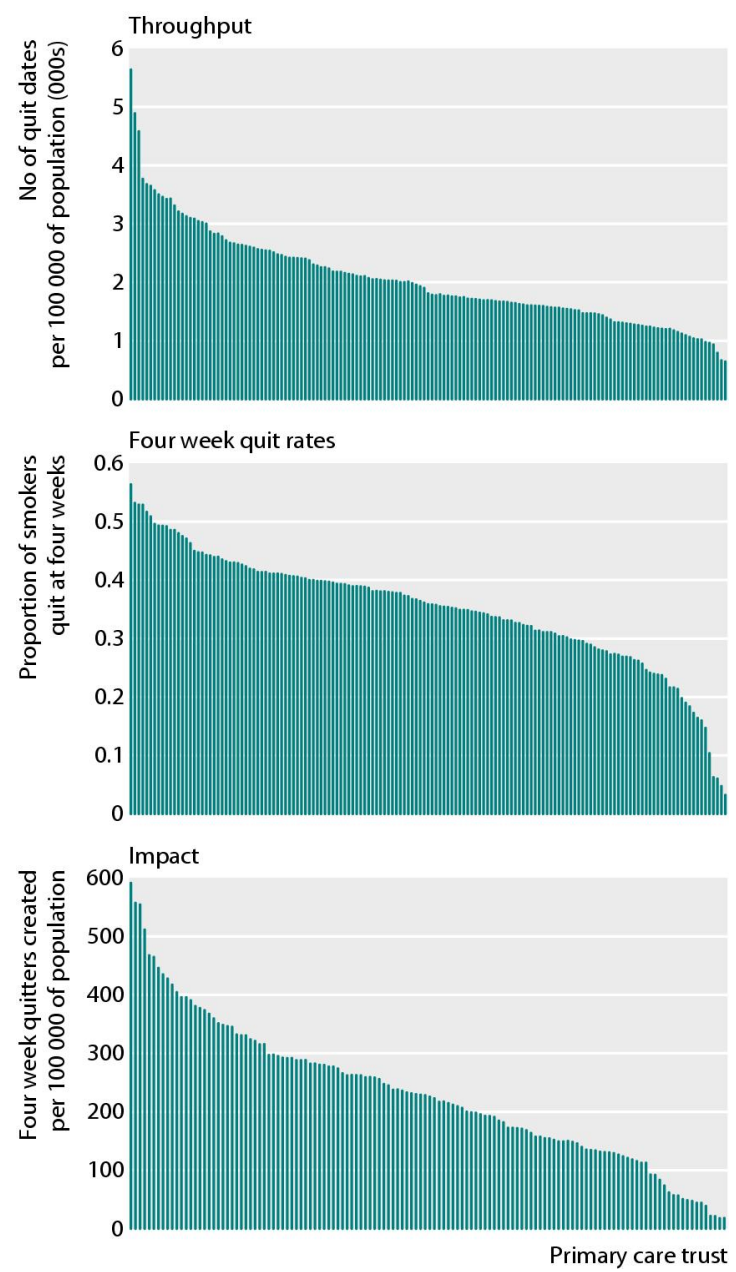

Fig 2 Throughput, four week quit rates, and impact of local stop smoking services in 2010/11 